\title{
Philosophiques
}

\section{Schnell, Alexander. Réflexion et spéculation. L'idéalisme transcendantal chez Fichte et Schelling. Grenoble, Jérôme Millon, collection Krisis, 2009, 232 p.}

\section{Manuel Roy}

Volume 38, numéro 1, printemps 2011

URI : https://id.erudit.org/iderudit/1005736ar

DOI : https://doi.org/10.7202/1005736ar

Aller au sommaire du numéro

Éditeur(s)

Société de philosophie du Québec

ISSN

0316-2923 (imprimé)

1492-1391 (numérique)

Découvrir la revue

Citer ce compte rendu

Roy, M. (2011). Compte rendu de [Schnell, Alexander. Réflexion et spéculation. L'idéalisme transcendantal chez Fichte et Schelling. Grenoble, Jérôme Millon, collection Krisis, 2009, 232 p.] Philosophiques, 38(1), 349-354.

https://doi.org/10.7202/1005736ar

Ce document est protégé par la loi sur le droit d'auteur. L'utilisation des services d'Érudit (y compris la reproduction) est assujettie à sa politique d'utilisation que vous pouvez consulter en ligne.

https://apropos.erudit.org/fr/usagers/politique-dutilisation/ 


\section{Schnell, Alexander. Réflexion et spéculation. L'idéalisme transcendantal chez Fichte et Schelling. Grenoble, Jérôme Millon, collection Krisis, 2009, $232 \mathrm{p}$.}

Avant tout spécialiste de la phénoménologie allemande et française ainsi que de Heidegger, Alexander Schnell s'intéresse depuis de nombreuses années à l'idéalisme allemand, et en particulier aux pensées de Fichte et de Schelling, notamment en vue de mettre en lumière les liens d'affiliation qui existent entre l'idéalisme transcendantal et la phénoménologie' ${ }^{1}$.

Dans son avant-dernier ouvrage, Réflexion et Spéculation. L'idéalisme transcendantal chez Fichte et Schelling, paru en 2009 chez Jérôme Millon, Schnell aborde la question de la différence entre les systèmes philosophiques de Fichte et de Schelling. S'il est nécessaire d'étudier à nouveau cette question initialement soulevée par Hegel en 1801 et déjà beaucoup débattue depuis, estime Schnell, c'est que le débat sur ce point s'est jusqu'à maintenant essentiellement déroulé dans l'ombre de la pensée de ce dernier et n'a fait que reproduire son jugement. Bien que Schnell ne prenne pas lui-même la peine d'expliquer en quel sens, il me paraît nécessaire, afin de mieux comprendre l'intention de ce dernier, de dire quelques mots à ce sujet.

Hegel, on le sait, s'est pour la première fois prononcé publiquement sur cette question dans la Differenzschrift, à une époque où Schelling passait encore généralement pour un simple porte-parole de Fichte. Une lecture attentive des œuvres de Fichte et de Schelling, expliquait alors Hegel dans ce texte, révèle que l'élève a dépassé le maître. En quel sens ?

Pour Fichte comme pour Schelling, il s'agit de mener à son terme la philosophie, conçue comme amour du savoir. Mais le savoir est conscience de l'identité de la représentation et de la réalité, du sujet et de l'objet. Achever la philosophie, c'est donc découvrir une voie d'accès à la conscience de cette identité.

Or Fichte, juge Hegel, ne parvient pas vraiment à cette conscience. Certes, la doctrine fichtéenne part de l'intuition intellectuelle dans laquelle le sujet est immédiatement saisi comme identique à l'objet. Cependant, cette intuition dans la perspective fichtéenne s'oppose à la conscience d'objet, avec laquelle elle doit être conciliée. La question de Fichte, dans la doctrine de la science, est alors la suivante: comment la conscience de l'identité du sujet et de l'objet peut-elle cohabiter avec la conscience de leur différence? Avec cette question, juge Hegel, la spéculation, c'est-à-dire l'activité réflexive de l'intelligence, qui au départ se posait elle-même comme absolue, "s'abandonne, elle abandonne son principe et ne revient pas en lui ${ }^{2} »$. Fichte conclut

1. Son dernier ouvrage, En deçà du sujet. Du temps dans la philosophie transcendantale allemande (Paris, PUF, 2010), explore ces liens d'affiliation de manière détaillée.

2. Hegel, La différence des systèmes philosophiques de Fichte et de Schelling, Paris, Vrin, 1986, p. 102. 
en effet, selon Hegel, que, puisqu'il y a conscience de la différence du sujet et de l'objet, la conscience ou l'affirmation de leur identité n'est pas d'ordre théorique, mais pratique. L'intelligence, dans la perspective fichtéenne, ne pose l'identité du sujet et de l'objet que parce que cette identité est exigée à titre de postulat de la conscience morale. En dernière analyse, donc, la conscience de l'identité du sujet et de l'objet n'est pas la conscience de ce qui est, mais de ce qui doit être (de ce qu'il me faut supposer comme étant) si je dois pouvoir m'affirmer comme être moral. Quant à la possibilité de la conscience de la réalité effective de cet état de chose, elle est renvoyée à l'infinie. Aussi Hegel caractérise-t-il l'idéalisme de Fichte comme subjectif, parce que l'affirmation fichtéenne de l'identité du sujet de l'objet repose exclusivement sur une nécessité morale dont la validité objective ne peut en aucun cas être confirmée.

Dans la perspective schellingienne, au contraire, note Hegel, la conscience de l'identité du sujet et de l'objet n'est pas opposée à la conscience de leur différence. Mais parce que Schelling admet en principe l'identité effective du sujet et de l'objet, saisie dans l'intuition intellectuelle, il en conclut que la pensée de leur différence est identique à la pensée de leur identité. C'est cette synthèse que le système schellingien vise à produire. C'est pourquoi la philosophie transcendantale, qui s'emploie à déduire la pensée de la différence du sujet et de l'objet à partir de la pensée de leur identité, doit être complétée dans la perspective schellingienne par une philosophie de la nature visant à faire ressortir le contraire, à savoir que la pensée de l'identité du sujet et de l'objet est impliquée dans la pensée de leur opposition. Autrement dit, Schelling soutient qu'on peut tout aussi bien penser la conscience subjective comme un accident de l'objet que la conscience objective comme un accident du sujet. C'est la raison pour laquelle l'apport de la doctrine schellingienne est caractérisé par Hegel comme idéalisme objectif, parce que Schelling développe une conséquence de l'idéalisme qui demeure occultée chez Fichte, à savoir qu'on ne saurait poser le principe de la matière dans l'intelligence sans poser également par le fait même le principe de l'intelligence dans la matière.

On voit comment cette compréhension de l'opposition Fichte/Schelling ouvre la voie à l'idéalisme absolu de Hegel, qui vient simplement synthétiser ces deux systèmes concurrents. En effet, Hegel est d'accord avec Schelling pour dire que la réalité finie constitue la manifestation de l'infini, que "tout phénomène partiel», comme l'écrit Bernard Gilson, "réintégré à sa place dans l'absolu, présente les caractères de l'absolu", ce qui "rend possible l'intuition du présent éternel dans le présent qui change ". Néanmoins, il nie avec Fichte la possibilité de l'intuition intellectuelle comme conscience pure de l'identité du sujet et de l'objet. Si tout être humain, pour

3. Bernard Gilson, «Présentation », in Hegel, La différence des systèmes philosophiques de Fichte et de Schelling, Paris, Vrin, 1986, p. 58. 
Hegel comme pour Fichte, tend à cette aperception de l'infini dans le fini, il ne l'atteint pas toujours, et lorsqu'il l'atteint il ne l'atteint jamais absolument: il demeure toujours un être humain. Comme l'écrit encore une fois B. Gilson: "l'idée humaine de Dieu [...], pour Hegel, reste celle d'un objet [...] qui ne devient jamais sujet, car le sujet ne peut jamais s'y reconnaître luimême ${ }^{4}$ ».

Or c'est avec cette interprétation hégélienne de Fichte et de Schelling, que d'aucuns ont qualifié d' "auto-congratulatoire" ", que Schnell entend rompre dans son étude: "L'idéalisme allemand", déclare-t-il, ne désigne pas la philosophie allemande post-kantienne en tant qu'elle culminerait dans le système hégélien, mais renvoie à différents systèmes - les plus connus d'entre eux étant ceux de Fichte, Schelling et Hegel - qui ne sont pas dans un rapport hiérarchique ( $4^{\mathrm{e}}$ de couv.)." Autrement dit, Schnell cherche à briser la conception dialectique de l'histoire de la pensée développée par Hegel, en vertu de laquelle les différents systèmes philosophiques, et en l'occurrence ceux de Fichte et de Schelling, doivent apparaître comme les divers moments, rationnellement nécessaires et s'articulant les uns par rapport aux autres selon la logique d'un dialogue assurant un progrès constant, de l'effort déployé par l'esprit dans le but de prendre conscience de ce qu'il est essentiellement. Car, selon Schnell, aussi paradoxal que cela puisse paraitre, il n'y a pour ainsi dire pas de dialogue entre Fichte et Schelling. Ou alors il s'agit d'un dialogue de sourd: Schelling ne vient pas simplement corriger et améliorer la doctrine fichtéenne, mais les pensées de Fichte et de Schelling représentent des "options philosophiques différentes, tellement différentes qu'elles exigent un choix (222)». Et un choix qui, selon Schnell, n'est pas de nature rationnelle: "Il ne s'agit pas, entre Fichte et Schelling, d'une opposition que l'on pourrait éventuellement trancher rationnellement, en usant "d'arguments", mais d'un rapport différent vis-àvis du RÉEL (222).» Bref, il n'y a pas de vérité une vers laquelle on progresserait à travers la réflexion de l'un, puis de l'autre; les positions exprimées par Fichte et Schelling ne sont pas susceptibles d'être synthétisées dans une doctrine plus englobante. Ni rapport ni continuité d'aucune sorte n'existent entre les philosophies de Fichte et de Schelling, mais plutôt rupture complète. Comprendre avec Schnell qu' "il est temps d'étudier les textes clés de Fichte et de Schelling pour eux-mêmes et non pas comme moments subordonnés du système hégélien ( $4^{\mathrm{e}}$ de couv.) ", c'est donc comprendre qu'il n'existe aucun terrain d'entente possible entre eux.

Ce diagnostic selon lequel les positions de Fichte et de Schelling, en dernière analyse, ne reposent pas sur des arguments - on en conviendra est assez étonnant en ce qui concerne des penseurs qui, plus qu'aucun autre

4. Ibid., p. 68.

5. Voir par exemple Wayne M. Martin, Idealism and Objectivity. Understanding Fichte's Jena Project, California, Stanford University Press, 1997, p. 11. 
auparavant, ont prétendu ne reconnaître aucune autorité hormis celle de la raison, qui ont cru pouvoir résoudre scientifiquement le problème philosophique et déterminer, avec une rigueur comparable à celle avec laquelle on procède en mathématiques, le fondement de toute réalité. Une telle lecture apparaît malgré tout vraisemblable, si l'on admet avec Schnell que «le point nodal de la querelle [...] entre Fichte et Schelling (avant Hegel) [...] se situe au niveau de la compréhension, de l'interprétation et de la réappropriation, par ces deux penseurs, de la philosophie transcendantale héritée de Kant", et "en particulier de la notion même de transcendantal (15)». Cerner ce qui unit et divise les deux philosophes, affirme Schnell, suppose une compréhension de l'interprétation "de la notion $d u$ transcendantal par laquelle ils s'inscrivent [...] dans la tradition kantienne (15)». Le conflit Fichte/Schelling reposerait alors en quelque sorte sur un simple malentendu, puisque, ayant compris différemment le problème soulevé par Kant, ceux-ci ne parleraient pas de la même chose.

Certes, la question de départ, superficiellement, chez Fichte et Schelling, paraît être la même. Il s'agit, explique Schnell, de la question des conditions de possibilité de la connaissance a priori. La question est de savoir ce qui fait d'une connaissance une connaissance, «ce qui la fonde et ce qui la légitime (18)». Les deux penseurs se demandent dans quelle mesure une représentation peut être considérée comme conforme à la réalité, comme étant objective. Jusqu'ici, Schnell suit Hegel. Cependant, il soutient que la manière dont Fichte et Schelling, chacun de son côté, répondent à cette question permet de constater qu'ils la comprennent différemment (16).

Fichte, pour sa part, comprend le problème originairement soulevé par Kant dans les termes suivants: la possibilité d'une certitude absolue suppose un principe premier à partir duquel il soit possible de procéder dans la démonstration. Or, en tant que premier, ce principe ne peut évidemment pas être démontré. Cependant, il ne doit pas non plus s'agir d'une simple hypothèse, sans quoi les propositions fondées sur ce principe ne seraient pas nécessaires et seraient exclues du domaine du savoir (45-46). La question de la possibilité de la connaissance, du point de vue de Fichte, est alors celle de l'«bypothéticité catégorique $(37,47)$ ", c'est-à-dire la question suivante: comment une proposition peut-elle être à la fois problématique et nécessaire (35) ? Ce problème, qui selon Schnell demeure le problème central de Fichte dans la totalité de l'œuvre, trouve également dans la perspective fichtéenne, dans la totalité de l'œuvre, une solution unique (21-22): c'est l'impératif moral trouvant son expression dans le concept de devoir qui constitue pour Fichte la synthèse de ces deux aspects apparemment contradictoires. "Fichte inscrit le pratique dans le théorique (48).» L'affirmation So soll es sein exprime que ce qui est simplement possible sur le plan théorique se trouve néanmoins admis comme certain pour des raisons morales. Le soll fait le pont entre l'idéalité et la réalité (47). Ainsi, le principe posé par Fichte au fondement de toute connaissance n'exprime pas ce qui est à proprement 
parler, mais ce qui doit être, c'est-à-dire ce dont il est nécessaire d'admettre la réalité, d'un point de vue moral ou pratique.

Chez Schelling, en revanche, le problème kantien est interprété autrement. Selon lui, il ne s'agit pas simplement de savoir comment une proposition indémontrable - c'est-à-dire constituant elle-même son propre fondement - peut être par ailleurs nécessaire. Poser le problème dans ces termes, estime Schelling, c'est s'en tenir à la pure forme de la connaissance. Comme le dit Schnell: "La différence fondamentale [entre les systèmes philosophiques de Fichte et de Schelling] concerne le reproche, que Schelling adresse à Fichte, d'après lequel la doctrine de la science serait purement formelle (219). » La question des conditions de possibilité de la connaissance ne concerne pas simplement la nature du sentiment de nécessité qui accompagne certaines de nos représentations. Ce qui caractérise les représentations que nous appelons des connaissances, ce n'est pas seulement qu'elles s'imposent à nous avec nécessité, comme le croit Fichte. Mais dans la connaissance, la représentation, c'est-à-dire l'agir de l'intellect, est pensé comme étant à la fois identique et différent de ce qui est représenté. En effet, nous pensons la connaissance comme une représentation adéquate (116), c'est-à-dire comme la représentation de quelque chose qui n'est pas représentation, mais à quoi la représentation est tout à fait semblable. Et, selon Schelling, c'est de ce double mouvement d'identité et de différence qu'il s'agit de rendre compte. Pour citer Schnell encore une fois: "Il ne s'agit [...] pas simplement de s'interroger sur la manière dont, en partant du Moi, ce même Moi peut rencontrer le Non-Moi. Schelling souligne bien plutôt un caractère du savoir qui n'a pas été considéré de façon suffisamment radicale, selon lui, dans la philosophie transcendantale - notamment kantienne et fichtéenne - à savoir la coïncidence réciproque (117). "C'est ce qui explique la cohabitation, chez Schelling, de deux systèmes: l'un - la philosophie transcendantale - qui vise à démontrer comment ce qui, par essence, n'est que représentation, peut être pensé comme existant de manière autonome, indépendamment de toute faculté de connaître; l'autre — la philosophie de la nature - qui vise à expliquer comment ce qui, par essence, échappe à la faculté de connaître, parvient tout de même à la représentation de soi (219220).

Cette manière d'envisager l'opposition Fichte/Schelling est-elle juste? On doit, à mon avis, convenir qu'elle est à tout le moins pertinente et défendable. Reste à savoir cependant si elle s'écarte vraiment, comme le prétend Schnell, de l'interprétation proposée par Hegel dans la Diffenrenzschrift. En ce qui concerne la différence des systèmes de Fichte et Schelling considérés en eux-mêmes, Schnell, me semble-t-il, reprend à peu de choses près la lecture hégélienne: Fichte aurait posé en principe l'identité du sujet et de l'objet comme postulat pratique; Schelling, l'identité effective du sujet et de l'objet. Schnell ne prend vraiment ses distances par rapport à Hegel qu'en ce qui concerne l'origine de cette différence, lorsqu'il affirme qu'elle vient d'une 
compréhension différente du transcendantal. Certes, Fichte et Schelling envisagent de manière distincte le problème hérité de Kant. Schnell l'a bien vu et, sur ce point, sa lecture est brillante. Mais cette différence de compréhension est-elle irréductible? Repose-t-elle simplement, comme le suggère Schnell, sur un choix existentiel s'effectuant sur le plan irrationnel? $\mathrm{Ou}$ n'est-il pas plutôt nécessaire d'admettre que cette différence de compréhension, à son tour, repose sur un jugement concernant la manière dont il convient d'envisager la notion de vérité ? Schelling critique la conception fichtéenne de la vérité, qu'il ne juge que partiellement juste. Pourquoi ce jugement ne pourrait-il pas faire l'objet d'une discussion rationnelle et argumentée? Pourquoi devrait-il dépendre exclusivement, comme le soutient Schnell - paraphrasant le fameux mot de Fichte — «de l'homme que l'on est (222)»?

MANUEL ROY

Université Humboldt Berlin

\section{Lindberg, Susanna. Heidegger contre Hegel. Les Irréconciliables, Paris, L'Harmattan, 2010, $222 \mathrm{p}$.}

Ce livre constitue sans conteste une des bonnes surprises de l'année 2010. L'auteure y réussit ni plus ni moins le coup double d'y éclairer Hegel par Heidegger, et Heidegger par Hegel. Grâce à une conception herméneutique novatrice du concept de dialogue mettant en jeu le conflit comme méthode philosophique, elle renouvelle tant le sens de l'existential du "Dasein» que celui de la finitude dans la dialectique.

La tâche à laquelle ce livre répond, à savoir mettre en dialogue Hegel et Heidegger, peut pourtant sembler peu originale. On ne compte plus les mises en relation de ces deux penseurs. Qui en douterait trouverait d'ailleurs dans l'ample bibliographie sur laquelle s'appuient les développements de cet ouvrage matière à se remettre en cause. Mais Susanna Lindberg évite avec brio les travers d'une stérile répétition des travaux déjà effectués en soumettant son sujet à une approche originale. Elle entend non seulement éviter de prendre parti pour l'un des philosophes au détriment de l'autre, mais aussi éviter de les comparer en adoptant un point de vue de surplomb. De telles démarches ou bien refuseraient le dialogue ou bien ne l'articuleraient qu'extérieurement. Contre ces démarches, il s'agit pour l'auteure de trouver les conditions d'un dialogue véritable entre les deux pensées en se faisant l'auxiliaire du dialogue qu'elles recèlent virtuellement. Ce qu'il importe de faire dialoguer, ce sont moins deux philosophes, qui d'ailleurs ne sont pas contemporains, que deux pensées. Pour ce faire, l'auteure réactive dans un premier temps chacune de ces pensées dans son nœud dialogique. En d'autres termes, elle montre comment chez Hegel et Heidegger s'envisage le dialogue. Loin d'un dialogique intersubjectif demandant la co-présence des parties en dia- 